\title{
Experimental Investigation of Water Hammer Phenomena in a Helical Coil Tube for Examination of Water Hammer Models
}

\author{
Taehoon Kim, Kyu Hyung Do, Byung-Il Choi, Yong-Shik Han \\ Korea Institute of Machinery \& Materials (KIMM) \\ 156, Gajeongbuk-ro, Yuseong-gu, Daejeon, 34103, Korea \\ thkim79@kimm.re.kr; kyudo@kimm.re.kr; cbisey@kimm.re.kr; yshan@kimm.re.kr
}

\section{Extended Abstract}

Since all flows will eventually be changed, either suddenly or gradually, all pipeline systems inevitably experience transient effects [1]. These effects can normally produce sudden increase in pressure in the pipeline systems. These phenomena are called water hammer. Water hammer phenomena in the pipeline systems can result in not only damage to equipment, but also possible injury to plant personnel. Due to these safe problems, it is important to predict water hammer in the pipeline systems. In this study, water hammer phenomena in a helical coil tube are experimentally investigated.

First of all, a friction factor is very important to estimate pressure propagation behaviour in water hammer prediction. Several models for the friction factors in a helical coil tube have been developed. However, there exists discrepancies between the models and there is no model for a friction factor of a helical coil tube in transition regime. Therefore, friction factors in a helical coil tube are experimentally obtained for laminar, transition, and turbulent regimes and compared with the previous models. Based on the comparison, the appropriate models are used for estimation of water hammer phenomena.

Then, experiments are performed for water hammer phenomena. Because pressure propagation in water hammer phenomena is very fast, a helical coil tube should be long in order to have time enough to measure the pressure propagation. So, the length of a helical coil tube is determined as $100 \mathrm{~m}$. For the implementation of sudden change of flows, a shutdown valve is located at the end of a helical coil tube. After the valve is suddenly closed, water hammer phenomena are occurred and the increased pressure wave travels along the tube. In order to measure the pressure propagation along the tube, pressure transducers with high frequency are installed every 10 meters along the tube. Experiments are performed as varying flow rate. As a result, the amplitude of pressure wave increases as increasing flow rate but the period of pressure wave is constant regardless to flow rate. Sometimes, the pressure in a pipeline drops to the vapour pressure at high flow rate. Then, cavitation is occurred in the tube. In this state, the amplitude of pressure wave is very large, it is not cyclic, and its period is not constant.

Finally, experimental results for water hammer in a helical coil tube are compared with the models developed by previous researchers. In this study, the water hammer models are considered because they have high accuracy compared to the other models [1]. The water hammer models and the method how to solve them are well explained in [2,3]. By comparison between experimental results and the models, it is shown that the unsteady friction coefficient model developed by Reddy et al. [4] corresponds well experimental results in this study. The results based on the other models have some deviations from the experimental results. In addition, when the cavitation is occurred during the water hammer phenomena, the pressure wave propagations are totally different from the water hammer models considered in this study. For this case, it is necessary to develop new models to predict pressure wave propagations under the cavitation.

\section{Acknowledgement}

This work was supported by the Korea Institute of Energy Technology Evaluation and Planning (KETEP) and the Ministry of Trade, Industry \& Energy (MOTIE) of the Republic of Korea (No. 20153030081190).

\section{References}

[1] B. S. Jung and B. Karney, "A practical overview of unsteady pipe flow modeling: from physics to numerical solutions," Urban Water J., vol. 14, no. 5, pp. 502-508, 2017. 
[2] M. H. Chaudhry, Applied Hydraulic Transients. 3rd ed., Springer, 1986.

[3] J. Vitkovsky, A. Bergant, A. Simpson, and M. Lambert, "Systematic evaluation of one-dimensional unsteady friction models in simple pipelines," J. Hydraulic Engineering, vol. 132, no. 7, pp. 696-708, 2006.

[4] H. P. Reddy, W. Silva-Araya, and M. H. Chaudhry, "Estimation of Decay Coefficients for Unsteady Friction for Instantaneous, Acceleration-Based Models,” J. Hydraulic Engineering, vol. 138, no. 3, pp. 260-271, 2012. 